\title{
A CLASS OF NONLINEAR DELAY EVOLUTION EQUATIONS WITH NONLOCAL INITIAL CONDITIONS
}

\author{
MONICA-DANA BURLICĂ AND DANIELA ROŞU
}

(Communicated by Yingfei Yi)

\begin{abstract}
We establish a sufficient condition for the existence, uniqueness and global uniform asymptotic stability of a $C^{0}$-solution for the nonlinear delay differential evolution equation$$
\begin{cases}u^{\prime}(t) \in A u(t)+f\left(t, u_{t}\right), & t \in \mathbb{R}_{+}, \\ u(t)=g(u)(t), & t \in[-\tau, 0],\end{cases}
$$

where $\tau>0, X$ is a real Banach space, $A$ is the infinitesimal generator of a nonlinear semigroup of contractions, $f: \mathbb{R}_{+} \times C([-\tau, 0] ; \overline{D(A)}) \rightarrow X$ is continuous and $g: C_{b}([-\tau,+\infty) ; \overline{D(A)}) \rightarrow C([-\tau, 0] ; \overline{D(A)})$ is nonexpansive.
\end{abstract}

\section{INTRODUCTION}

The goal of this paper is to prove an existence and uniform asymptotic stability result of a $C^{0}$-solution for the nonlinear delay differential evolution equation with nonlocal initial data

$$
\begin{cases}u^{\prime}(t) \in A u(t)+f\left(t, u_{t}\right), & t \in \mathbb{R}_{+}, \\ u(t)=g(u)(t), & t \in[-\tau, 0] .\end{cases}
$$

Here $A: D(A) \subseteq X \rightsquigarrow X$ is an $m$-dissipative operator in the real (infinite dimensional) Banach space $X, \tau>0$ is arbitrarily fixed, $f: \mathbb{R}_{+} \times C([-\tau, 0] ; \overline{D(A)}) \rightarrow X$ is continuous and $g: C_{b}([-\tau,+\infty) ; \overline{D(A)}) \rightarrow C([-\tau, 0] ; \overline{D(A)})$ is nonexpansive. If $I$ is an interval, $C_{b}(I ; X)$ denotes the space of all bounded and continuous functions from $I$, equipped with the sup-norm $\|\cdot\|_{C_{b}(I ; X)}$, while $C_{b}(I ; \overline{D(A)})$ denotes the closed subset in $C_{b}(I ; X)$ consisting of all elements $u \in C_{b}(I ; X)$ satisfying $u(t) \in \overline{D(A)}$ for each $t \in I$. Further, $C([a, b] ; X)$ stands for the space of all continuous functions from $[a, b]$ to $X$ endowed with the sup-norm $\|\cdot\|_{C([a, b] ; X)}$ and $C([a, b] ; \overline{D(A)})$ is the closed subset of $C([a, b] ; X)$ containing all $u \in C([a, b] ; X)$ with $u(t) \in \overline{D(A)}$ for each $t \in[a, b]$. As usual, if $u \in C([-\tau,+\infty) ; X)$ and $t \in[0,+\infty), u_{t} \in C([-\tau, 0] ; X)$ is defined by $u_{t}(s):=u(t+s)$ for each $s \in[-\tau, 0]$.

Received by the editors June 14, 2012 and, in revised form, July 30, 2012.

2010 Mathematics Subject Classification. Primary 34K05, 34K13, 34K20, 34K30, 35K55, $35 \mathrm{~K} 65,47 \mathrm{H} 05$.

Key words and phrases. Differential delay evolution equation, nonlocal delay initial condition, periodic solutions, metric fixed point arguments, nonresonance condition, nonlinear parabolic equations.

This work was supported by a grant of the Romanian National Authority for Scientific Research, CNCS-UEFISCDI, project number PN-II-ID-PCE-2011-3-0052. 
Remark 1.1. As observed by Hale [9, Section 2, p. 11], in the case $A=0$ and $X=\mathbb{R}^{n}$, and also in our case, the class of evolution differential-difference problems of the form

$$
\left\{\begin{array}{l}
u^{\prime}(t) \in A u(t)+F\left(t, u(t), u\left(t-\tau_{1}\right), \ldots, u\left(t-\tau_{n}\right)\right), \quad t \in \mathbb{R}_{+}, \\
u(t)=g(u)(t), \quad t \in[-\tau, 0],
\end{array}\right.
$$

where $0<\tau_{1}<\tau_{2}<\cdots<\tau_{n} \leq \tau$ are some intermediate delays and the forcing term $F: \mathbb{R}_{+} \times[\overline{D(A)}]^{n+1} \rightarrow X$ is continuous or Lipschitz, falls into our general framework. Indeed, if we denote by $\delta(s-\theta)$ the Dirac delta concentrated at $s=-\theta$, we have

$$
\begin{aligned}
& F\left(t, u(t), u\left(t-\tau_{1}\right), \ldots, u\left(t-\tau_{n}\right)\right) \\
& \quad=F\left(t, \delta(s-0) u_{t}, \delta\left(s-\tau_{1}\right) u_{t}, \ldots, \delta\left(s-\tau_{n}\right) u_{t}\right):=f\left(t, u_{t}\right) .
\end{aligned}
$$

Since $\delta(s-\theta)$ is nonexpansive from $C([-\tau, 0] ; X)$ to $X$, we easily conclude that the function $f: \mathbb{R}_{+} \times C([-\tau, 0] ; \overline{D(A)}) \rightarrow X$, defined as above, is continuous or Lipschitz whenever $F$ is continuous or Lipschitz with respect to its last $n+1$ arguments. Accordingly, our main result applies also to problems of the form (1.2), but not only.

Differential-difference evolution equations of the specific form (1.2) were recently studied by Vrabie 21 under the hypotheses that $A$ generates a nonlinear compact semigroup which decays exponentially, $F$ is jointly continuous and the class of those $g: C_{b}([-\tau,+\infty) ; \overline{D(A)}) \rightarrow C([-\tau, 0] ; \overline{D(A)})$, allowed in (1.2), is general enough to contain elements of the form

$$
g(u)(t)=\int_{\tau}^{+\infty} \mathcal{N}(u(t+\theta)) d \mu(\theta)
$$

for each $u \in C_{b}([-\tau,+\infty) ; \overline{D(A)})$ and $t \in[-\tau, 0]$. In (1.3), $\mathcal{N}: X \rightarrow X$ is a (possible nonlinear) nonexpansive operator and $\mu$ is a $\sigma$-finite and complete measure on $[\tau,+\infty)$, satisfying supp $\mu=[\delta,+\infty), \delta \geq \tau$ and $\mu([\tau,+\infty))=1$. In turn, by obvious particular choices of $\mathcal{N}$ and $\mu$, the class of functions defined by (1.3) includes the following three important subclasses:

(i) $g(u)(t)=u(2 \pi+t), t \in[-\tau, 0](2 \pi$-periodicity condition);

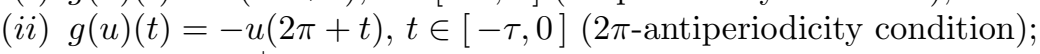

(iii) $g(u)(t)=\int_{\tau}^{+\infty} k(\theta) u(t+\theta) d \theta, t \in[-\tau, 0]$, where $k \in L^{1}([\tau,+\infty) ; \mathbb{R})$ and

$$
\int_{\tau}^{+\infty}|k(\theta)| d \theta=1 \text { (mean condition). }
$$

Vrabie 21 has also shown that one can get rid of the compactness of the semigroup if we assume, in addition, that $F$ is Lipschitz with respect to its last $n+1$ arguments and there exists $a>0$ such that $\|g(u)-g(v)\|_{C([-\tau, 0] ; X)} \leq$ $\|u-v\|_{C_{b}([a,+\infty) ; X)}$ for all $u, v \in C_{b}([-\tau,+\infty) ; X)$, a condition which corresponds to supp $\mu=[\delta,+\infty)$ with $\delta>\tau$ in (1.3).

Our main goal is to extend Theorem 3.5 in [21] from problems of the form (1.2) to problems of the form (1.1) under the very same general hypotheses on $A$ and on $g$ imposed by [21]. We notice that the main results in [21] generalize in several directions those of Li [12. More precisely, 12 analyzes the semilinear periodic 
case, i.e., when $g$ is of the form $(i)$ above, in a Hilbert space $H$, by assuming that $A: D(A) \subseteq H \rightarrow H$ is a linear operator which generates an analytic, compact semigroup which decays exponentially and $F$ is Lipschitz with respect to the last $n+1$ arguments.

Related results concerning nondelayed equations of the form (1.1) with nonlocal initial conditions where previously obtained by Byszewski [4, Garcia and Reich 8 and Paicu and Vrabie [15]. For some mathematical models described by evolution equations subjected to nonlocal initial conditions, see Deng [7] and McKibben [13, Section 10.2, pp. 394-398].

For periodic problems without delay related to our main results, see Caşcaval and Vrabie [5], Hirano [11, Paicu [14] and Vrabie [16]. As far as antiperiodic problems are concerned, see Aizicovici, Pavel and Vrabie [1. For a periodic, semilinear problem with infinite delay, see the recent paper of Henríquez and Lizama 10.

A multi-valued version of (1.2), i.e., for $F$ a multi-function, was considered by Vrabie [19, while similar results in the multi-valued case of (1.1) were obtained by Vrabie [20] and 22] under the additional hypothesis that $A$ generates a compact semigroup.

This paper is organized into 5 sections. In Section 2 we briefly review some concepts and results referring to evolutions governed by $m$-dissipative operators. In Section 3 we state the main result, i.e. Theorem 3.1 and one important consequence, i.e. Theorem 3.2, while in Section 4 we prove the above mentioned theorems. In Section 5, as applications of our abstract theory, we obtain two existence results for nonlinear parabolic problems with delay, subject either to general nonlocal initial conditions or to periodic conditions.

\section{Preliminaries}

The reader is expected to be familiar with the basic theory concerning $m$ dissipative operators and nonlinear evolution equations in Banach spaces. For details, we refer the reader to Barbu [2] and Vrabie [17. As far as functional differential equations with delay are concerned, we refer to [9]. For the sake of clarity, we recall some concepts and results we will frequently use in the sequel.

Let $X$ be a real Banach space with norm $\|\cdot\|$. Let $x, y \in X$ and $h \in \mathbb{R} \backslash\{0\}$. We denote

$$
[x, y]_{h}:=\frac{1}{h}(\|x+h y\|-\|x\|)
$$

and we recall that there exists

$$
\lim _{h \downarrow 0}[x, y]_{h}=\inf \left\{[x, y]_{h} ; h>0\right\}:=[x, y]_{+} .
$$

Remark 2.1. One may easily see that for each $x, y \in X$ and $\alpha>0$, we have

(i) $[\alpha x, y]_{+}=[x, y]_{+}$,

(ii) $\left|[x, y]_{+}\right| \leq\|y\|$.

For other remarkable properties of the mapping $(x, y) \mapsto[x, y]_{+}$, see $[2$, Proposition 3.7, p. 103].

An operator $A: D(A) \subseteq X \rightsquigarrow X$ is called dissipative if for each $x_{i} \in D(A)$ and $y_{i} \in A x_{i}, i=1,2$, we have

$$
\left[x_{1}-x_{2}, y_{2}-y_{1}\right]_{+} \geq 0 \text {. }
$$


It is called $m$-dissipative if it is dissipative and, in addition, $R(I-\lambda A)=X$, for each $\lambda>0$.

Let $f \in L^{1}(a, b ; X)$ and let us consider the evolution equation

$$
u^{\prime}(t) \in A u(t)+f(t) .
$$

A function $u:[a, b] \rightarrow \overline{D(A)}$ is called $a C^{0}$-solution, or integral solution of (2.1) on $[a, b]$, if $u \in C([a, b] ; X)$ and it satisfies

$$
\|u(t)-x\| \leq\|u(s)-x\|+\int_{s}^{t}[u(\tau)-x, f(\tau)+y]_{+} d \tau
$$

for each $x \in D(A), y \in A x$ and $a \leq s \leq t \leq b$.

Theorem 2.1. Let $\omega>0$ and let $A: D(A) \subseteq X \rightsquigarrow X$ be an m-dissipative operator such that $A+\omega I$ is dissipative. Then, for each $\xi \in \overline{D(A)}$ and $f \in L^{1}(a, b ; X)$, there exists a unique $C^{0}$-solution of (2.1) on $[a, b]$ which satisfies $u(a)=\xi$. If $f, g \in L^{1}(a, b ; X)$ and $u, v$ are two $C^{0}$-solutions of (2.1) corresponding to $f$ and $g$ respectively, then

$$
\|u(t)-v(t)\| \leq e^{-\omega(t-s)}\|u(s)-v(s)\|+\int_{s}^{t} e^{-\omega(t-\theta)}\|f(\theta)-g(\theta)\| d \theta
$$

for each $a \leq s \leq t \leq b$.

In particular, if $x \in D(A)$ and $y \in A x$, we have

$$
\|u(t)-x\| \leq e^{-\omega(t-s)}\|u(s)-x\|+\int_{s}^{t} e^{-\omega(t-\theta)}\|f(\theta)+y\| d \theta
$$

for each $a \leq s \leq t \leq b$.

See [2, Theorem 4.1, p. 130].

Let $\xi \in X, \tau \in[a, b)$ and $f \in L^{1}(a, b ; X)$. Thereafter, $u(\cdot, \tau, \xi, f)$ denotes the unique $C^{0}$-solution $v:[\tau, b] \rightarrow \overline{D(A)}$ of the problem (2.1) which satisfies the initial condition $v(\tau)=\xi$. We denote by $\{S(t): \overline{D(A)} \rightarrow \overline{D(A)}, t \geq 0\}$ the semigroup of nonexpansive mappings generated by $A$ on $\overline{D(A)}$ in the sense of Crandall and Liggett [6]. Then

for each $\xi \in X$ and $t \geq 0$.

$$
S(t) \xi=u(t, 0, \xi, 0)
$$

\section{The MAIN RESUlts}

Our main assumptions on $A, f$ and $g$ are listed below.

$\left(H_{A}\right)$ the operator $A: D(A) \subseteq X \rightsquigarrow X$ is $m$-dissipative, $0 \in D(A), 0 \in A 0$ and there exists $\omega>0$ such that $A+\omega I$ is dissipative;

$\left(H_{f}\right)$ the function $f: \mathbb{R}_{+} \times C([-\tau, 0] ; \overline{D(A)}) \rightarrow X$ is continuous and satisfies the following:

$\left(f_{1}\right)$ there exists $\ell>0$ such that

$$
\|f(t, v)-f(t, \widetilde{v})\| \leq \ell\|v-\widetilde{v}\|_{C([-\tau, 0] ; X)}
$$

for each $t \in \mathbb{R}_{+}$and $v, \widetilde{v} \in C([-\tau, 0] ; \overline{D(A)})$;

$\left(f_{2}\right)$ there exists $m>0$ such that

$$
\|f(t, 0)\| \leq m
$$

for each $t \in[0,+\infty)$; 
$\left(H_{c}\right)$ the constants $\ell, \tau>0$ and $\omega>0$ satisfy the nonresonance condition

$$
\ell<\omega ;
$$

$\left(H_{g}\right)$ the function $g: C_{b}([-\tau,+\infty) ; \overline{D(A)}) \rightarrow C([-\tau, 0] ; \overline{D(A)})$ satisfies that:

$\left(g_{1}\right)$ for each $u \in C_{b}([-\tau,+\infty) ; \overline{D(A)})$, we have

$$
\|g(u)\|_{C([-\tau, 0] ; X)} \leq\|u\|_{C_{b}([0,+\infty) ; X)} ;
$$

$\left(g_{2}\right)$ there exists $a>0$ such that for each $u, v \in C_{b}([-\tau,+\infty) ; \overline{D(A)})$, we have

$$
\|g(u)-g(v)\|_{C([-\tau, 0] ; X)} \leq\|u-v\|_{C_{b}([a,+\infty) ; X)} .
$$

Remark 3.1. We notice that whenever the function $g$ is defined as in $(i)-(i i i)$ in the Introduction and $\tau<2 \pi$, then $g$ satisfies $\left(g_{1}\right),\left(g_{2}\right)$. If the support of the measure $\mu$ is in $(\tau,+\infty)$, then the function $g$ given by (1.3) satisfies $\left(g_{1}\right)$ and $\left(g_{2}\right)$. Moreover, if $g$ satisfies $\left(g_{1}\right)$ and $\left(g_{2}\right)$, then it depends only on the restriction of $u$ to $[0,+\infty)$, i.e., $g(u)=g(\widetilde{u})$ whenever $u(t)=\widetilde{u}(t)$ for each $t \in[0,+\infty)$. Consequently, in this case, $g$ can be defined only on $C_{b}([0,+\infty) ; \overline{D(A)})$ and not on the whole $C_{b}([-\tau,+\infty) ; \overline{D(A)})$. However, due to the fact that we are looking for $C^{0}$-solutions of the problem (1.1) which belong to $C_{b}([-\tau,+\infty) ; \overline{D(A)})$, we prefer to consider that $g$ is defined on the latter set.

Our main result, which is an extension of Theorem 3.5 in [21], is:

Theorem 3.1. If $\left(H_{A}\right),\left(H_{f}\right),\left(H_{g}\right)$ and $\left(H_{c}\right)$ are satisfied, then (1.1) has a unique $C^{0}$-solution, $u \in C_{b}([-\tau,+\infty) ; \overline{D(A)})$, satisfying

$$
\|u\|_{C_{b}([-\tau,+\infty) ; X)} \leq \frac{m}{\omega-\ell} .
$$

If instead of $\left(H_{c}\right)$ the stronger condition $\ell e^{\omega \tau}<\omega$ is satisfied, then $u$ is globally asymptotically stable.

We recall that $u$ is globally asymptotically stable if for each $\varphi \in C([-\tau, 0] ; \overline{D(A)})$ the unique $C^{0}$-solution $v \in C([-\tau,+\infty) ; \overline{D(A)})$ of the problem

satisfies

$$
\begin{cases}v^{\prime}(t) \in A v(t)+f\left(t, v_{t}\right), & t \in[0,+\infty), \\ v(t)=\varphi(t), & t \in[-\tau, 0],\end{cases}
$$

$$
\lim _{t \rightarrow+\infty}\|u(t)-v(t)\|=0 .
$$

If we choose $\tau \in(0,2 \pi)$ and $g(u)(t)=u(t+2 \pi), t \in[-\tau, 0]$, we obtain the next result for the existence of the solution of the periodic problem

$$
\begin{cases}u^{\prime}(t) \in A u(t)+f\left(t, u_{t}\right), & t \in \mathbb{R}, \\ u(t)=u(t+2 \pi), & t \in \mathbb{R} .\end{cases}
$$

More precisely, we have:

Theorem 3.2. If $\left(H_{A}\right),\left(H_{f}\right)$ and $\left(H_{c}\right)$ are satisfied and $f: \mathbb{R} \times C([-\tau, 0] ; \overline{D(A)}) \rightarrow$ $X$ is $2 \pi$-periodic with respect to its first argument, then (3.2) has a unique $2 \pi$ periodic $C^{0}$-solution, $u: \mathbb{R} \rightarrow \overline{D(A)}$, satisfying

$$
\|u\|_{C([0,2 \pi] ; X)} \leq \frac{m}{\omega-\ell} .
$$

If, in addition, $\ell e^{\omega \tau}<\omega$, then $u$ is globally asymptotically stable. 


\section{Proofs of Theorems 3.1 and 3.2}

The proof, inspired from the proof of Theorem 3.5 in [21], is based on:

Lemma 4.1. If $\left(H_{A}\right),\left(H_{f}\right),\left(H_{g}\right)$ and $\left(H_{c}\right)$ are satisfied, then, for each $v \in$ $C_{b}([-\tau,+\infty) ; \overline{D(A)})$, the problem

$$
\begin{cases}u^{\prime}(t) \in A u(t)+f\left(t, v_{t}\right), & t \in \mathbb{R}_{+}, \\ u(t)=g(u)(t), & t \in[-\tau, 0],\end{cases}
$$

has a unique $C^{0}$-solution $u \in C_{b}([-\tau,+\infty) ; \overline{D(A)})$.

Proof. Let $v \in C_{b}([-\tau,+\infty) ; \overline{D(A)})$ be arbitrary but fixed. Let us further consider $w \in C_{b}([-\tau,+\infty) ; \overline{D(A)})$ and the auxiliary problem

$$
\begin{cases}u^{\prime}(t) \in A u(t)+f\left(t, v_{t}\right), & t \in \mathbb{R}_{+}, \\ u(t)=g(w)(t), & t \in[-\tau, 0] .\end{cases}
$$

Clearly, (4.5) has a unique $C^{0}$-solution $u \in C_{b}([-\tau,+\infty) ; \overline{D(A)})$. Since the existence is immediate, we have merely to check that $u$ is bounded. Indeed, if $t \geq 0$, using (2.3) we deduce that

$$
\begin{gathered}
\|u(t)\| \leq e^{-\omega t}\|g(w)(0)\|+\int_{0}^{t} e^{-\omega(t-s)}\left\|f\left(s, v_{s}\right)\right\| d s \\
\leq\|g(w)\|_{C([-\tau, 0] ; X)}+\int_{0}^{t} e^{-\omega(t-s)}\left(\left\|f\left(s, v_{s}\right)-f(s, 0)\right\|+\|f(s, 0)\|\right) d s .
\end{gathered}
$$

From $\left(H_{f}\right)$ and $\left(g_{1}\right)$ we get

$$
\|u(t)\| \leq\|w\|_{C_{b}([0,+\infty) ; X)}+\frac{1}{\omega}\left(\ell\|v\|_{C_{b}([-\tau,+\infty) ; X)}+m\right),
$$

for each $t \geq 0$. For $t \in[-\tau, 0)$, by $\left(g_{1}\right)$, we have

$$
\|u(t)\| \leq\|g(w)\|_{C([-\tau, 0] ; X)} \leq\|w\|_{C_{b}([0,+\infty) ; X)} .
$$

So, we can define the operator $T: C_{b}([-\tau,+\infty) ; \overline{D(A)}) \rightarrow C_{b}([-\tau,+\infty) ; \overline{D(A)})$ by $T(w)=u$, where $u \in C_{b}([-\tau,+\infty) ; \overline{D(A)})$ is the unique $C^{0}$-solution of the problem (4.5). Obviously, (4.4) has a unique $C^{0}$-solution if and only if $T$ has a unique fixed point. So, to complete the proof, it suffices to show that $T^{2}$ is a strict contraction. To this aim, let $w, z \in C_{b}([-\tau,+\infty) ; \overline{D(A)})$. We have

$$
\|T w-T z\|_{C_{b}([a,+\infty) ; X)} \leq e^{-\omega a}\|w-z\|_{C_{b}([a,+\infty) ; X)} .
$$

Indeed, if $t \geq a$ using (2.3) and $\left(g_{2}\right)$, we obtain

$$
\begin{gathered}
\|(T w)(t)-(T z)(t)\| \leq e^{-\omega t}\|g(w)(0)-g(z)(0)\| \\
\leq e^{-\omega t}\|g(w)-g(z)\|_{C([-\tau, 0] ; X)} \leq e^{-\omega a}\|w-z\|_{C_{b}([a,+\infty) ; X)} .
\end{gathered}
$$

Now we prove that

$$
\left\|T^{2} w-T^{2} z\right\|_{C_{b}([-\tau,+\infty) ; X)} \leq e^{-\omega a}\|w-z\|_{C_{b}([-\tau,+\infty) ; X)} .
$$

First, let $t \geq 0$. By $\left(g_{2}\right)$ and (4.6), we have

$$
\begin{aligned}
& \left\|\left(T^{2} w\right)(t)-\left(T^{2} z\right)(t)\right\| \leq e^{-\omega t}\|(g(T w))(0)-(g(T z))(0)\| \\
\leq & e^{-\omega t}\|g(T w)-g(T z)\|_{C([-\tau, 0] ; X)} \leq\|T w-T z\|_{C_{b}([a,+\infty) ; X)} \\
\leq & e^{-\omega a}\|w-z\|_{C_{b}([a,+\infty) ; X)} \leq e^{-\omega a}\|w-z\|_{C_{b}([-\tau,+\infty) ; X) .}
\end{aligned}
$$


Second, if $t \in[-\tau, 0)$, using the same inequalities, i.e., $\left(g_{2}\right)$ and (4.6), we get $\left\|\left(T^{2} w\right)(t)-\left(T^{2} z\right)(t)\right\|=\|(g(T w))(t)-(g(T z))(t)\| \leq\|g(T w)-g(T z)\|_{C([-\tau, 0] ; X)}$ $\leq\|T w-T z\|_{C_{b}([a,+\infty) ; X)} \leq e^{-\omega a}\|w-z\|_{C_{b}([-\tau,+\infty) ; X)}$.

So, for each $t \in[-\tau,+\infty)$ we have

$$
\left\|\left(T^{2} w\right)(t)-\left(T^{2} z\right)(t)\right\| \leq e^{-\omega a}\|w-z\|_{C_{b}([-\tau,+\infty) ; X)},
$$

which implies (4.7). But (4.7) shows that $T^{2}$ is a contraction of the constant $e^{-\omega a}$. So, $T$ has a unique fixed point $u$ which is a $C^{0}$-solution of problem (4.4). This completes the proof.

Lemma 4.2. Let $x, y \in C_{b}\left([-\tau,+\infty) ; \mathbb{R}_{+}\right)$and let us assume that there exist $a>0$, $\omega>0, \ell>0$ and $m \geq 0$ such that

$$
x(t) \leq\|x\|_{C_{b}([a,+\infty) ; \mathbb{R})}
$$

for each $t \in[-\tau, 0]$ and

$$
x(t) \leq e^{-\omega t}\|x\|_{C_{b}([a,+\infty) ; \mathbb{R})}+\frac{\ell}{\omega}\left(1-e^{-\omega t}\right)\left(\|y\|_{C_{b}([-\tau,+\infty) ; \mathbb{R})}+\frac{m}{\ell}\right)
$$

for each $t \in[0,+\infty)$. Then

$$
\|x\|_{C_{b}([-\tau,+\infty) ; \mathbb{R})} \leq \frac{\ell}{\omega}\left(\|y\|_{C_{b}([-\tau,+\infty) ; \mathbb{R})}+\frac{m}{\ell}\right) .
$$

Proof. We prove first that

$$
\|x\|_{C_{b}([a,+\infty) ; \mathbb{R})} \leq \frac{\ell}{\omega}\left(\|y\|_{C_{b}([-\tau,+\infty) ; \mathbb{R})}+\frac{m}{\ell}\right) .
$$

We distinguish between two possible complementary cases.

Case 1. There exists $\tilde{t} \in[a,+\infty)$ such that

$$
\|x\|_{C_{b}([a,+\infty) ; \mathbb{R})}=x(\widetilde{t}) .
$$

Setting $t=\widetilde{t}$ in (4.9), we get

$$
\|x\|_{C_{b}([a,+\infty) ; \mathbb{R})} \leq e^{-\omega \widetilde{t}}\|x\|_{C_{b}([a,+\infty) ; \mathbb{R})}+\frac{\ell}{\omega}\left(1-e^{-\omega \widetilde{t}}\right)\left(\|y\|_{C_{b}([-\tau,+\infty) ; \mathbb{R})}+\frac{m}{\ell}\right)
$$

and, since $\widetilde{t} \geq a>0$, we deduce (4.11).

Case 2. If there is no $\tilde{t} \in[a,+\infty)$ such that (4.12) holds true, then there does exist at least one sequence $\left(t_{k}\right)_{k} \subseteq[a,+\infty)$ with $\lim _{k} t_{k}=+\infty$ and

$$
\|x\|_{C_{b}([a,+\infty) ; \mathbb{R})}=\lim _{k} x\left(t_{k}\right) .
$$

Setting $t=t_{k}$ in (4.9) and letting $k \rightarrow+\infty$, we again obtain (4.11).

Now, let us prove (4.10).

Here, we distinguish between three possible cases.

Case 1. There exists $\bar{t} \in[-\tau, 0]$ such that

$$
\|x\|_{C_{b}([-\tau,+\infty) ; \mathbb{R})}=x(\bar{t}) .
$$

By (4.8), we get

and thus

$$
x(\bar{t}) \leq\|x\|_{C_{b}([a,+\infty) ; \mathbb{R})}
$$

and by (4.11), we deduce (4.10).

$$
\|x\|_{C_{b}([-\tau,+\infty) ; \mathbb{R})}=\|x\|_{C_{b}([a,+\infty) ; \mathbb{R})},
$$


Case 2. There exists $\bar{t}>0$ such that

$$
\|x\|_{C_{b}([-\tau,+\infty) ; \mathbb{R})}=x(\bar{t}) .
$$

Using (4.9), we conclude that

$\|x\|_{C_{b}([-\tau,+\infty) ; \mathbb{R})} \leq e^{-\omega \bar{t}}\|x\|_{C_{b}([-\tau,+\infty) ; \mathbb{R})}+\frac{\ell}{\omega}\left(1-e^{-\omega \bar{t}}\right)\left(\|y\|_{C_{b}([-\tau,+\infty) ; \mathbb{R})}+\frac{m}{\ell}\right)$.

Since $\bar{t}>0$, we easily get (4.10).

Case 3. If, for each $t \in[-\tau,+\infty)$,

$$
x(t)<\|x\|_{C_{b}([-\tau,+\infty) ; \mathbb{R})},
$$

then there exists $\left(t_{k}\right)_{k}$ with $\lim _{k} t_{k}=+\infty$ such that

$$
\lim _{k} x\left(t_{k}\right)=\|x\|_{C_{b}([-\tau,+\infty) ; \mathbb{R})} .
$$

Setting $t=t_{k}$ in (4.9) and letting $k \rightarrow+\infty$, we obtain (4.10).

So, in all possible cases, $x$ and $y$ satisfy (4.10), and this completes the proof.

The next lemma is perhaps known, but since we did not find any reference to it in the form we need later, we include it with a complete proof.

Lemma 4.3. Let $y:[-\tau,+\infty) \rightarrow \mathbb{R}_{+}$and $\alpha_{0}, \beta:[0,+\infty) \rightarrow \mathbb{R}_{+}$be continuous functions with $\alpha_{0}$ nondecreasing. If

$$
y(t) \leq \alpha_{0}(t)+\int_{0}^{t} \beta(s)\left\|y_{s}\right\|_{C([-\tau, 0]: \mathbb{R})} d s
$$

for each $t \in[0,+\infty)$, then

$$
y(t) \leq \alpha(t)+\int_{0}^{t} \alpha(s) \beta(s) e^{\int_{s}^{t} \beta(\sigma) d \sigma} d s
$$

for each $t \in[0,+\infty)$, where

$$
\alpha(t):=\left\|y_{0}\right\|_{C([-\tau, 0] ; \mathbb{R})}+\alpha_{0}(t)
$$

for each $t \in[0,+\infty)$.

Proof. Let us denote by $z:[-\tau,+\infty) \rightarrow \mathbb{R}_{+}$the function

$$
z(t):=\sup \left\{\left\|y_{s}\right\|_{C([-\tau, 0] ; \mathbb{R})} ; s \in[0, t]\right\}=\sup \{y(\theta) ; \theta \in[-\tau, t]\} .
$$

Clearly $y(t) \leq z(t)$ for each $t \in[0,+\infty)$. Since $y$ is continuous, for each $t \in[0,+\infty)$ there exists $s_{t} \in[-\tau, t]$ such that $z(t)=y\left(s_{t}\right)$.

We distinguish between two cases.

Case 1. If $s_{t} \in[-\tau, 0]$ and $y(s)<z(s)$ for each $s \in(0, t]$, then

$$
z(t)=y\left(s_{t}\right) \leq\left\|y_{0}\right\|_{C([-\tau, 0] ; \mathbb{R})} \leq \alpha(t)+\int_{0}^{t} \beta(s) z(s) d s .
$$

Case 2. If $s_{t} \in(0, t]$, then

$$
\begin{gathered}
z(t)=y\left(s_{t}\right) \leq \alpha_{0}\left(s_{t}\right)+\int_{0}^{s_{t}} \beta(\sigma)\left\|y_{\sigma}\right\|_{C([-\tau, 0] ; \mathbb{R})} d \sigma \\
\leq \alpha_{0}(t)+\left\|y_{0}\right\|_{C([-\tau, 0] ; \mathbb{R})}+\int_{0}^{t} \beta(\sigma) z(\sigma) d \sigma=\alpha(t)+\int_{0}^{t} \beta(\sigma) z(\sigma) d \sigma .
\end{gathered}
$$


So, 4.15) holds true for each $t \in[0,+\infty)$. From Bellman's Inequality (see Vrabie [18, Problem 1.16, p. 48]) we get

$$
z(t) \leq \alpha(t)+\int_{0}^{t} \alpha(s) \beta(s) e^{\int_{s}^{t} \beta(\sigma) d \sigma} d s
$$

for each $t \in[0,+\infty)$. Since $y(t) \leq z(t)$ for each $t \in[0,+\infty)$, the inequality above implies (4.14). The proof is complete.

\subsection{Proof of Theorem 3.1.}

Proof. The proof is based on a fixed point argument. Namely, let us define the operator $Q: C_{b}([-\tau,+\infty) ; \overline{D(A)}) \rightarrow C_{b}([-\tau,+\infty) ; \overline{D(A)})$ by $Q(v)=u$, where $u \in C_{b}([-\tau,+\infty) ; \overline{D(A)})$ is the unique $C^{0}$-solution of the problem

$$
\begin{cases}u^{\prime}(t) \in A u(t)+f\left(t, v_{t}\right), & t \in \mathbb{R}_{+}, \\ u(t)=g(u)(t), & t \in[-\tau, 0] .\end{cases}
$$

To complete the proof, it would suffice to show that $Q$ is a strict contraction. To this aim, let $v, w \in C_{b}([-\tau,+\infty) ; \overline{D(A)})$ be arbitrary and let us observe that for each $t \geq 0$, from (2.3) and $\left(f_{1}\right)$ we get

$$
\begin{gathered}
\|Q(v)(t)-Q(w)(t)\| \leq e^{-\omega t}\|Q(v)(0)-Q(w)(0)\|+\frac{\ell}{\omega}\left(1-e^{-\omega t}\right) \sup _{s \in[-\tau, t]}\left\|v_{s}-w_{s}\right\| \\
\leq e^{-\omega t}\|g(Q(v))(0)-g(Q(w))(0)\|+\frac{\ell}{\omega}\left(1-e^{-\omega t}\right)\|v-w\|_{C_{b}([-\tau,+\infty) ; X)} \\
\leq e^{-\omega t}\|g(Q(v))-g(Q(w))\|_{C([-\tau, 0] ; X)}+\frac{\ell}{\omega}\left(1-e^{-\omega t}\right)\|v-w\|_{C_{b}([-\tau,+\infty) ; X) .}
\end{gathered}
$$

Thanks to $\left(g_{2}\right)$, we conclude that for each $t \geq 0$, we have

$$
\begin{gathered}
\|Q(v)(t)-Q(w)(t)\| \\
\leq e^{-\omega t}\|Q(v)-Q(w)\|_{C_{b}([a,+\infty) ; X)}+\frac{\ell}{\omega}\left(1-e^{-\omega t}\right)\|v-w\|_{C_{b}([-\tau,+\infty) ; X)} .
\end{gathered}
$$

Since, by $\left(g_{2}\right)$, we have

$$
\|Q(v)(t)-Q(w)(t)\| \leq\|Q(v)-Q(w)\|_{C_{b}([a,+\infty) ; X)}
$$

for each $t \in[-\tau, 0]$, denoting by $x(t)=\|Q(v)(t)-Q(w)(t)\|, y(t)=\|v(t)-w(t)\|$ for $t \in[-\tau,+\infty), \omega>0, \ell>0$ as above and $m=0$, we have merely to observe that we are in the hypotheses of Lemma 4.2. Then, we conclude that

$$
\|Q(v)-Q(w)\|_{C_{b}([-\tau,+\infty) ; X)} \leq \frac{\ell}{\omega}\|v-w\|_{C_{b}([-\tau,+\infty) ; X)},
$$

which, in view of $\left(H_{c}\right)$, i.e., $\ell<\omega$, shows that $Q$ is a strict contraction.

In order to prove the inequality (3.1), let us observe first that by $\left(g_{1}\right)$ we have $g(0)=0$, while from $\left(g_{2}\right)$ we get

$$
\|g(u)\|_{C([-\tau, 0] ; X)} \leq\|u\|_{C_{b}([a,+\infty) ; X)}
$$

for each $u \in C_{b}([-\tau,+\infty) ; \overline{D(A)})$.

Since $0 \in A 0$, from (2.3) in Theorem 2.1, we have

$$
\|u(t)\| \leq e^{-\omega t}\|g(u)\|_{C([-\tau, 0] ; X)}+\int_{0}^{t} e^{-\omega(t-s)}\left(\left\|f\left(s, u_{s}\right)-f(s, 0)\right\|+\|f(s, 0)\|\right) d s,
$$


for each $t \geq 0$. Next, using $\left(H_{f}\right)$ and (4.18), we deduce that

$$
\|u(t)\| \leq e^{-\omega t}\|u\|_{C_{b}([a,+\infty) ; X)}+\frac{\ell}{\omega}\left(1-e^{-\omega t}\right)\left(\|u\|_{C_{b}([-\tau,+\infty) ; X)}+\frac{m}{\ell}\right) .
$$

Since, by the initial nonlocal condition and $\left(g_{2}\right)$ we have

$$
\|u(t)\| \leq\|u\|_{C_{b}([a,+\infty) ; X)}
$$

for each $t \in[-\tau, 0]$, it follows that all the hypotheses of Lemma 4.2 are satisfied by $x(t)=y(t)=\|u(t)\|$ and $\omega>0, \ell>0$ and $m>0$ as above. So

$$
\|u\|_{C_{b}([-\tau,+\infty) ; X)} \leq \frac{\ell}{\omega}\left(\|u\|_{C_{b}([-\tau,+\infty) ; X)}+\frac{m}{\ell}\right),
$$

from which we get 3.1 ).

Finally, let $u \in C_{b}([-\tau,+\infty) ; \overline{D(A)})$ be the unique $C^{0}$-solution of (1.1), let $\varphi \in C([-\tau, 0] ; \overline{D(A)})$ be arbitrary but fixed, and let $v \in C_{b}([-\tau,+\infty) ; \overline{D(A)})$ be the unique $C^{0}$-solution of the problem

$$
\begin{cases}v^{\prime}(t) \in A v(t)+f\left(t, v_{t}\right), & t \in[0,+\infty), \\ v(t)=\varphi(t), & t \in[-\tau, 0] .\end{cases}
$$

In view of (2.2) and of $\left(f_{1}\right)$ in $\left(H_{f}\right)$, we have

$$
\begin{aligned}
\| u(t) & -v(t)\left\|\leq e^{-\omega t}\right\| u(0)-v(0)\left\|+\int_{0}^{t} e^{-\omega(t-s)}\right\| f\left(s, u_{s}\right)-f\left(s, v_{s}\right) \| d s \\
& \leq e^{-\omega t}\|u(0)-v(0)\|+\int_{0}^{t} \ell e^{-\omega(t-s)}\left\|u_{s}-v_{s}\right\|_{C([-\tau, 0] ; X)} d s
\end{aligned}
$$

for each $t \geq 0$. Multiplying both sides by $e^{\omega t}$ and observing that

$$
e^{\omega s}\left\|u_{s}-v_{s}\right\|_{C([-\tau, 0] ; X)} \leq e^{\omega \tau} \sup _{\theta \in[-\tau, 0]}\left\{e^{\omega(s+\theta)}\|u(s+\theta)-v(s+\theta)\|\right\},
$$

we deduce that

$$
e^{\omega t}\|u(t)-v(t)\| \leq\|u(0)-v(0)\|+\int_{0}^{t} \ell e^{\omega \tau} \sup _{\theta \in[-\tau, 0]}\left\{e^{\omega(s+\theta)}\|u(s+\theta)-v(s+\theta)\|\right\} d s
$$

for each $t \geq 0$. From Lemma 4.3 with

$$
\begin{cases}y(t)=e^{\omega t}\|u(t)-v(t)\|, & t \in[-\tau,+\infty), \\ \alpha_{0}(t)=\|u(0)-v(0)\|:=c, & t \in[0,+\infty), \\ \beta(t)=\ell e^{\omega \tau}:=b, & t \in[0,+\infty),\end{cases}
$$

we get

$$
e^{\omega t}\|u(t)-v(t)\| \leq c+\|u-v\|_{C([-\tau, 0] ; X)}+b\left(c+\|u-v\|_{C([-\tau, 0] ; X)}\right) \int_{0}^{t} e^{b(t-s)} d s
$$

for each $t \geq 0$. Accordingly, we have

$$
\|u(t)-v(t)\| \leq\left(c+\|u-v\|_{C([-\tau, 0] ; X)}\right) e^{-(\omega-b) t}
$$

for each $t \geq 0$. Since $b=\ell e^{\omega \tau}<\omega$, this shows that

$$
\lim _{t \rightarrow+\infty}\|u(t)-v(t)\|=0,
$$

which means that $u$ is globally asymptotically stable. This completes the proof. 


\subsection{Proof of Theorem $\mathbf{3 . 2}$.}

Proof. We have only to observe that (3.2) can be equivalently rewritten as (1.1) where $g: C_{b}([-\tau,+\infty) ; \overline{D(A)}) \rightarrow C([-\tau, 0] ; \overline{D(A)})$ is defined by

$$
g(u)(t)=u(2 \pi+t)
$$

for each $u \in C_{b}([-\tau,+\infty) ; \overline{D(A)})$ and each $t \in[-\tau, 0]$. Let $u \in C_{b}([-\tau,+\infty) ; \overline{D(A)})$ be arbitrary but fixed. Then, there exists $t_{u} \in[-\tau, 0]$ such that

$$
\|g(u)\|_{C([-\tau, 0] ; X)}=\left\|g(u)\left(t_{u}\right)\right\|=\left\|u\left(2 \pi+t_{u}\right)\right\|
$$

and, since $2 \pi+t_{u} \geq 2 \pi-\tau>0$, we deduce that

$$
\|g(u)\|_{C([-\tau, 0] ; X)} \leq\|u\|_{C_{b}([0,+\infty) ; X)},
$$

which shows that $g$ satisfies $\left(g_{1}\right)$ in $\left(H_{g}\right)$.

To check $\left(g_{2}\right)$, let $u, v \in C_{b}([-\tau,+\infty) ; \overline{D(A)})$ be arbitrary but fixed and observe that there exists $t_{u, v} \in[-\tau, 0]$ such that

$\|g(u)-g(v)\|_{C([-\tau, 0] ; X)}=\left\|g(u)\left(t_{u, v}\right)-g(v)\left(t_{u, v}\right)\right\|=\left\|u\left(2 \pi+t_{u, v}\right)-v\left(2 \pi+t_{u, v}\right)\right\|$.

But $2 \pi+t_{u, v} \geq 2 \pi-\tau=a>0$, and therefore

$$
\|g(u)-g(v)\|_{C([-\tau, 0] ; X)} \leq\|u-v\|_{C_{b}([a,+\infty) ; X)} .
$$

So, $g$ satisfies $\left(g_{2}\right)$ in $\left(H_{g}\right)$ too. Thus we are in the hypotheses of Theorem 3.1 . Since (3.3) follows from (3.1) in conjunction with the periodicity condition, this completes the proof.

\section{EXAmples}

Let $\Omega$ be a nonempty, bounded domain in $\mathbb{R}^{n}, n \geq 2$, with $C^{1}$ boundary $\Gamma$, let $\tau>0, \omega>0$ and let $\Delta$ be the Laplace operator in the sense of distributions over $\Omega$. Let $\varphi: D(\varphi) \subseteq \mathbb{R} \rightsquigarrow \mathbb{R}$ be maximal-monotone, let $f: \mathbb{R}_{+} \times C\left([-\tau, 0] ; L^{1}(\Omega)\right) \rightarrow$ $L^{1}(\Omega)$ be continuous, let $\mathcal{N}: L^{1}(\Omega) \rightarrow L^{1}(\Omega)$ be a (possible nonlinear) nonexpansive operator and $\mu$ a $\sigma$-finite and complete measure on $[\tau,+\infty)$ with supp $\mu=[\delta,+\infty)$, $\delta>\tau$. We consider the following problem with nonlocal initial conditions:

$$
\begin{cases}\frac{\partial u}{\partial t}(t, x)=\Delta \varphi(u(t, x))-\omega u(t, x)+f\left(t, u_{t}\right)(x), & (t, x) \in \mathbb{R}_{+} \times \Omega, \\ \varphi(u(t, x))=0, & (t, x) \in \mathbb{R}_{+} \times \Gamma, \\ u(t, x)=\int_{\tau}^{+\infty} \mathcal{N}(u(t+\theta, \cdot))(x) d \mu(\theta), & (t, x) \in[-\tau, 0] \times \Omega .\end{cases}
$$

If $u: \Omega \rightarrow D(\varphi)$, we denote

$$
\mathcal{S}_{\varphi}(u)=\left\{v \in L^{1}(\Omega) ; v(x) \in \varphi(u(x)) \text {, a.e. for } x \in \Omega\right\} .
$$

We recall the following result of Brezis and Strauss [3].

Theorem 5.1. Let $\Omega$ be a nonempty, bounded domain in $\mathbb{R}^{n}, n \geq 2$, with $C^{1}$ boundary $\Gamma$ and let $\varphi: D(\varphi) \subseteq \mathbb{R} \rightsquigarrow \mathbb{R}$ be maximal-monotone with $0 \in \varphi(0)$. Then the operator $\Delta \varphi: D(\Delta \varphi) \subseteq L^{1}(\Omega) \rightsquigarrow L^{1}(\Omega)$, defined by

$$
\begin{aligned}
& D(\Delta \varphi)=\left\{u \in L^{1}(\Omega) ; \exists v \in \mathcal{S}_{\varphi}(u) \cap W_{0}^{1,1}(\Omega), \Delta v \in L^{1}(\Omega)\right\}, \\
& \Delta \varphi(u)=\left\{\Delta v ; v \in \mathcal{S}_{\varphi}(u) \cap W_{0}^{1,1}(\Omega)\right\} \cap L^{1}(\Omega)
\end{aligned}
$$

for each $u \in D(\Delta \varphi)$, is m-dissipative on $L^{1}(\Omega)$. 
Theorem 5.2. Let $\Omega$ be a nonempty, bounded domain in $\mathbb{R}^{n}$ with $C^{1}$ boundary $\Gamma$, let $\tau>0, \omega>0$ and let $\varphi: D(\varphi) \subseteq \mathbb{R} \rightsquigarrow \mathbb{R}$ be maximal-monotone with $0 \in \varphi(0)$. Let $f: \mathbb{R}_{+} \times C\left([-\tau, 0] ; L^{1}(\Omega)\right) \rightarrow L^{1}(\Omega)$ be continuous, let $\mathcal{N}: L^{1}(\Omega) \rightarrow L^{1}(\Omega)$ be $a$ (possible nonlinear) operator with $\mathcal{N}(0)=0$ and let $\mu$ be a $\sigma$-finite and complete measure on $[\tau,+\infty)$ with supp $\mu=[\delta,+\infty), \delta>\tau$. If the following hypotheses are satisfied:

$\left(F_{1}\right)$ there exists $\ell \in(0, \omega)$ such that

$$
\|f(t, v)-f(t, \widetilde{v})\|_{L^{1}(\Omega)} \leq \ell\|v-\widetilde{v}\|_{C\left([-\tau, 0] ; L^{1}(\Omega)\right)}
$$

for each $t \in \mathbb{R}_{+}$and each $v, \widetilde{v} \in C\left([-\tau, 0] ; L^{1}(\Omega)\right)$,

$\left(F_{2}\right)$ there exists $m>0$ such that for each $t \in \mathbb{R}_{+}$we have

$$
\|f(t, 0)\|_{L^{1}(\Omega)} \leq m,
$$

$(N)$ for each $u, \widetilde{u} \in L^{1}(\Omega)$ we have

$$
\|\mathcal{N}(u)-\mathcal{N}(\widetilde{u})\|_{L^{1}(\Omega)} \leq\|u-\widetilde{u}\|_{L^{1}(\Omega)},
$$

( $\mu$ ) $\mu([\tau,+\infty))=1$,

then the problem (5.20) has a unique $C^{0}$-solution, $u \in C_{b}\left([-\tau,+\infty) ; L^{1}(\Omega)\right)$, satisfying

$$
\|u\|_{C_{b}\left([-\tau,+\infty) ; L^{1}(\Omega)\right)} \leq \frac{m}{\omega-\ell} .
$$

If, in addition, instead of $\ell \in(0, \omega)$, the stronger condition $\ell e^{\omega \tau}<\omega$ is satisfied, then $u$ is globally asymptotically stable.

Proof. We will apply Theorem 3.1 with $X=L^{1}(\Omega) ; A: D(A) \subseteq L^{1}(\Omega) \rightsquigarrow L^{1}(\Omega)$, defined by $D(A)=D(\Delta \varphi) ; \quad A u=\Delta \varphi(u)-\omega u$ for each $u \in D(A)$, where $\Delta \varphi$ is as in Theorem 5.1] $f: \mathbb{R}_{+} \times C\left([-\tau, 0] ; L^{1}(\Omega)\right) \rightarrow L^{1}(\Omega)$ as above and $g$ : $C_{b}\left([-\tau,+\infty) ; L^{1}(\Omega)\right) \rightarrow C\left([-\tau, 0] ; L^{1}(\Omega)\right)$ given by (1.3) $)$, i.e.,

$$
(g(u)(t))(x)=\int_{\tau}^{+\infty} \mathcal{N}(u(t+\theta)(\cdot))(x) d \mu(\theta)
$$

for each $u \in C\left([-\tau, 0] ; L^{1}(\Omega)\right), t \in[-\tau, 0]$ and a.e. for $x \in \Omega$.

In view of Theorem 5.1, the operator $A$ satisfies $\left(H_{A}\right)$ in Theorem 3.1. From $\left(F_{1}\right)$ and $\left(F_{2}\right)$ we deduce that $f$ satisfies $\left(H_{f}\right)$. As $\mathcal{N}(0)=0$, from $(N)$ we conclude that $\|\mathcal{N}(u)\|_{L^{1}(\Omega)} \leq\|u\|_{L^{1}(\Omega)}$ which, together with $(\mu)$, shows that $g$ satisfies $\left(g_{1}\right)$. If we consider $a=\delta-\tau>0$, where supp $\mu=[\delta,+\infty)$, the nonexpansivity condition on $\mathcal{N}$ proves that $g$ also satisfies $\left(g_{2}\right)$. So, the problem (5.20) can be rewritten as an abstract problem of the form (1.1) satisfying the hypotheses of Theorem 3.1. whence the conclusion of Theorem $[5.2$.

Remark 5.1. We indicate two important examples of forcing terms $f$ defined by starting from a real function $\tilde{f}: \mathbb{R} \times \Omega \times \mathbb{R} \rightarrow \mathbb{R}$ and satisfying the hypotheses $\left(F_{1}\right)$ and $\left(F_{2}\right)$.

(i) $f(t, u)(x):=\tilde{f}(t, x, u(-\tau, x))$ for each $t \in \mathbb{R}_{+}$, each $u \in C\left(\left[-\tau, L^{1}(\Omega)\right)\right.$ and a.e. for $x \in \Omega$. In this case, $f\left(t, u_{t}\right)(x)=\widetilde{f}(t, x, u(t-\tau, x))$ is the usual delay difference forcing term.

(ii) $f(t, u)(x):=\tilde{f}\left(t, x, \int_{-\tau}^{0} k(s) u(s, x) d s\right)$, which represents the neutral type forcing term. 
It is easy to check that whenever $\tilde{f}$ is continuous and there exist $\tilde{\ell}>0$ and $\widetilde{m}>0$ such that $|\widetilde{f}(t, x, u)-\widetilde{f}(t, x, v)| \leq \widetilde{\ell}|u-v|$ and $|\widetilde{f}(t, x, 0)| \leq \widetilde{m}$ for each $t \in \mathbb{R}_{+}$, each $u, v \in \mathbb{R}$ and each $x \in \Omega$, then $f$, defined as in $(i)$, satisfies $\left(F_{1}\right)$ and $\left(F_{2}\right)$ with $\ell=\tilde{\ell}$ and $m=\widetilde{m}$. Furthermore, if $\tilde{f}$ is as above and $k \in L^{1}(-\tau, 0 ; \mathbb{R})$, then $f$, defined as in $(i i)$, satisfies $\left(F_{1}\right)$ and $\left(F_{2}\right)$ with $\ell=\widetilde{\ell}\|k\|_{L^{1}(-\tau, 0 ; \mathbb{R})}^{-1}$ and $m=\widetilde{m}\|k\|_{L^{1}(-\tau, 0 ; \mathbb{R})}^{-1}$.

As an application of Theorem 3.2, we consider the following nonlinear delay parabolic equation subjected to periodic conditions:

$$
\begin{cases}\frac{\partial u}{\partial t}(t, x)=\Delta \varphi(u(t, x))-\omega u(t, x)+f\left(t, u_{t}\right)(x), & (t, x) \in \mathbb{R} \times \Omega \\ \varphi(u(t, x))=0, & (t, x) \in \mathbb{R} \times \Gamma \\ u(t, x)=u(t+2 \pi, x), & (t, x) \in \mathbb{R} \times \Omega .\end{cases}
$$

Theorem 5.3. Let $\Omega$ be a nonempty, bounded domain in $\mathbb{R}^{n}$ with $C^{1}$ boundary $\Gamma$, let $\omega>0$ and let $\varphi: D(\varphi) \subseteq \mathbb{R} \rightsquigarrow \mathbb{R}$ be a maximal-monotone operator with $0 \in \varphi(0)$. Let $f: \mathbb{R}_{+} \times C\left([-\tau, 0] ; L^{1}(\Omega)\right) \rightarrow L^{1}(\Omega)$ be continuous and $2 \pi$-periodic with respect to its first argument. If $2 \pi>\tau$ and $f$ satisfies $\left(F_{1}\right)$ and $\left(F_{2}\right)$ in Theorem 5.2 , then the problem (5.21) has a unique $C^{0}$-solution $u \in C\left(\mathbb{R} ; L^{1}(\Omega)\right)$. If, in addition, instead of $\ell \in(0, \omega)$, the stronger condition $\ell e^{\omega \tau}<\omega$ is satisfied, then $u$ is globally asymptotically stable.

Remark 5.2. It should be emphasized that, as mentioned in 17, Remark 2.7.1, p. 72], under the hypotheses of both Theorems 5.2 and 5.3, the semigroup generated by $A$ on $L^{1}(\Omega)$ is not necessarily compact. Therefore, Theorems 5.2 and 5.3 cannot be obtained as single-valued corollaries of the main results in [20] and [22].

\section{ACKNOWLEDGEMENT}

The authors would like to express their warmest thanks to the referees for a careful reading of the paper and for the suggestions and critical comments that contributed to the improvement of the presentation.

\section{REFERENCES}

[1] S. Aizicovici, N. H. Pavel, and I. I. Vrabie, Anti-periodic solutions to strongly nonlinear evolution equations in Hilbert spaces, An. Ştiinţ. Univ. Al. I. Cuza Iaşi. Mat. (N.S.) 44 (1998), no. 2, 227-234 (2000). Dedicated to Professor C. Corduneanu on the occasion of his 70th birthday. MR 1783202 (2001f:34118)

[2] Viorel Barbu, Nonlinear differential equations of monotone types in Banach spaces, Springer Monographs in Mathematics, Springer, New York, 2010. MR2582280 (2011d:34001)

[3] Haïm Brézis and Walter A. Strauss, Semi-linear second-order elliptic equations in $L^{1}$, J. Math. Soc. Japan 25 (1973), 565-590. MR0336050 (49 \#826)

[4] Ludwik Byszewski, Theorems about the existence and uniqueness of solutions of a semilinear evolution nonlocal Cauchy problem, J. Math. Anal. Appl. 162 (1991), no. 2, 494-505, DOI 10.1016/0022-247X(91)90164-U. MR.1137634 (92m:35005)

[5] Radu Caşcaval and Ioan I. Vrabie, Existence of periodic solutions for a class of nonlinear evolution equations, Rev. Mat. Univ. Complut. Madrid 7 (1994), no. 2, 325-338. MR.1297518 (95i:34113)

[6] M. G. Crandall and T. M. Liggett, Generation of semi-groups of nonlinear transformations on general Banach spaces, Amer. J. Math. 93 (1971), 265-298. MR0287357 (44 \#4563)

[7] Keng Deng, Exponential decay of solutions of semilinear parabolic equations with nonlocal initial conditions, J. Math. Anal. Appl. 179 (1993), no. 2, 630-637, DOI 10.1006/jmaa.1993.1373. MR 1249842(95a:35076) 
[8] Jesús García-Falset and Simeon Reich, Integral solutions to a class of nonlocal evolution equations, Commun. Contemp. Math. 12 (2010), no. 6, 1031-1054, DOI 10.1142/S021919971000410X. MR2748284 (2012b:34163)

[9] Jack Hale, Theory of functional differential equations, 2nd ed., Applied Mathematical Sciences, Vol. 3, Springer-Verlag, New York, 1977. MR0508721 (58 \#22904)

[10] Hernán R. Henríquez and Carlos Lizama, Periodic solutions of abstract functional differential equations with infinite delay, Nonlinear Anal. 75 (2012), no. 4, 2016-2023, DOI 10.1016/j.na.2011.10.002. MR.2870895(2012m:34128)

[11] Norimichi Hirano, Existence of periodic solutions for nonlinear evolution equations in Hilbert spaces, Proc. Amer. Math. Soc. 120 (1994), no. 1, 185-192, DOI 10.2307/2160185. MR 1174494 (94b:34087)

[12] Yongxiang Li, Existence and asymptotic stability of periodic solution for evolution equations with delays, J. Funct. Anal. 261 (2011), no. 5, 1309-1324, DOI 10.1016/j.jfa.2011.05.001. MR2807101 (2012d:34177)

[13] M. McKibben, Discovering Evolution Equations with Applications. Vol. I. Deterministic Models, Appl. Math. Nonlinear Sci. Ser., Chapman \& Hall/CRC, 2011.

[14] Angela Paicu, Periodic solutions for a class of nonlinear evolution equations in Banach spaces, An. Ştiinţ. Univ. Al. I. Cuza Iaşi. Mat. (N.S.) 55 (2009), no. 1, 107-118. MR2510715 (2010e:35169)

[15] Angela Paicu and Ioan I. Vrabie, A class of nonlinear evolution equations subjected to nonlocal initial conditions, Nonlinear Anal. 72 (2010), no. 11, 4091-4100, DOI 10.1016/j.na.2010.01.041. MR2606769 (2011b:34171)

[16] Ioan I. Vrabie, Periodic solutions for nonlinear evolution equations in a Banach space, Proc. Amer. Math. Soc. 109 (1990), no. 3, 653-661, DOI 10.2307/2048204. MR.1015686 (90k:34080)

[17] I. I. Vrabie, Compactness methods for nonlinear evolutions, 2nd ed., with a foreword by A. Pazy, Pitman Monographs and Surveys in Pure and Applied Mathematics, vol. 75, Longman Scientific \& Technical, Harlow, 1995. MR.1375237 (96k:47116)

[18] Ioan I. Vrabie, Differential equations. An introduction to basic concepts, results and applications, 2nd ed., World Scientific Publishing Co. Pte. Ltd., Hackensack, NJ, 2011. MR2839525 (2012e:34001)

[19] Ioan I. Vrabie, Existence for nonlinear evolution inclusions with nonlocal retarded initial conditions, Nonlinear Anal. 74 (2011), no. 18, 7047-7060, DOI 10.1016/j.na.2011.07.025. MR:2833693 (2012g:34147)

[20] Ioan I. Vrabie, Existence in the large for nonlinear delay evolution inclusions with nonlocal initial conditions, J. Funct. Anal. 262 (2012), no. 4, 1363-1391, DOI 10.1016/j.jfa.2011.11.006. MR2873844

[21] Ioan I. Vrabie, Nonlinear retarded evolution equations with nonlocal initial conditions, Dynam. Systems Appl. 21 (2012), no. 2-3, 417-439. MR2918389

[22] Ioan I. Vrabie, Global solutions for nonlinear delay evolution inclusions with nonlocal initial conditions, Set-Valued Var. Anal. 20 (2012), no. 3, 477-497, DOI 10.1007/s11228-012-0203-6. MR2949638

Department of Mathematics and Informatics, "G. Asachi" Technical University, Bvd.

CAROL I, no. 11 A, IAŞi, 700506, Romania

E-mail address: monicaburlica@yahoo.com

Department of Mathematics and Informatics, "G. Asachi" Technical University, Bvd.

CARol I, NO. 11 A, IAŞI, 700506, Romania

E-mail address: rosudaniela100@yahoo.com 